\title{
The Predictive Value of Autoantibody Spectrum on Organ Damage in Patients With Systemic Lupus Erythematosus
}

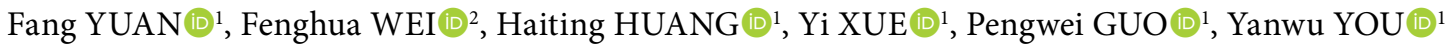 \\ ${ }^{1}$ Department of Nephrology, Affiliated Hospital of Youjiang Medical University for Nationalities, Baise, China \\ ${ }^{2}$ Affiliated Hospital of Youjiang Medical University for Nationalities, Outpatients, Baise, China
}

\begin{abstract}
Objectives: This study aims to investigate the positive detection rate and predictive value of autoantibodies, including anti-double stranded deoxyribonucleic acid (anti-dsDNA) antibodies, anti-histone antibodies (AHAs), anti-ribosomal (anti-Rib) P antibodies, anti-Smith (anti-Sm) antibodies, anti-U1 ribonucleoprotein (anti-U1RNP) antibodies, anti-Sjögren's syndrome type A antibodies and anti-Sjögren's syndrome type B antibodies, on organ damage in patients with systemic lupus erythematous (SLE).

Patients and methods: A total of 225 patients with SLE (37 males, 188 females; mean age $37.4 \pm 15.9$ years; range, 7 to 80 years) were evaluated retrospectively. Statistical analysis was performed to obtain the positive detection rate of autoantibodies and to investigate the predictive value.

Results: There were statistically significant differences of positive anti-dsDNA antibodies in renal damage, photosensitization, hematological abnormalities and serositis $(p<0.05)$ and a statistically significant difference of positive AHAs in photosensitization $(p<0.05)$. There was statistically significant difference of positive anti-U1RNP antibodies in renal damage $(p<0.05)$. There were also statistically significant differences of positive anti-Smith antibodies in renal damage, arthritis, photosensitization, oral ulcers, hematological abnormalities and serositis $(p<0.05)$ and of positive anti-Rib antibodies in renal damage, arthritis, photosensitization, malar rash, hematological abnormalities and serositis $(p<0.05)$. However, there were no statistically significant differences of positive anti-Sjögren's syndrome type B antibodies and anti-Sjögren's syndrome type A antibodies in renal damage, arthritis, malar rash, neuropsychiatric disorders, hematological abnormalities and serositis ( $p>0.05)$.

Conclusion: Autoantibody spectrum is an important serological basis for SLE diagnosis. There are differences in the autoantibodies distribution of SLE patients with different organ damage, suggesting a certain clinical value for prediction of organ damage in SLE.

Keywords: Autoantibodies, organ damage, systemic lupus erythematous.
\end{abstract}

Systemic lupus erythematosus (SLE) is a multi-organ autoimmune disease with a broad spectrum of clinical presentations and complicated pathogenesis. ${ }^{1}$ The SLE prevalence rates ranged from 40 to 122 per $100,000 .^{2}$ SLE can affect virtually any organ and can involve the skin, joints, heart, lungs, kidneys and central nervous system. It is characterized by excessive production of various autoantibodies that are then deposited in tissues. Some antibodies are associated with certain organ damage, e.g., anti-double stranded deoxyribonucleic acid (anti-dsDNA) antibodies are associated with nephritis. ${ }^{3-5}$ Anti-Smith (anti-Sm) antibodies have shown associations with constitutional symptoms, ${ }^{6}$ lupus nephritis ${ }^{3,5}$ and diseases of the central nervous system. ${ }^{7}$ However, certain autoantibody profiles that correlate with organ damage in SLE have not been well-studied. Therefore, in this study, we aimed to investigate the positive detection rate and predictive value of various autoantibodies, including anti-dsDNA antibodies, anti-histone antibodies

Received: April 13, 2018 Accepted: May 15, 2018 Published online: August 16, 2018

Correspondence: Yanwu You, MD. Department of Nephrology, Affiliated Hospital of Youjiang Medical University for Nationalities, 533000 Baise, China. Tel: 07762805530 e-mail:youyanwu@163.com Arch Rheumatol 2019;34(2):157-165. 
(AHAs), anti-ribosomal (anti-Rib) $\mathrm{P}$ antibodies, anti-Sm antibodies, anti-U1 ribonucleoprotein (anti-U1RNP) antibodies, anti-Sjögren's syndrome type A (anti-SSA) antibodies and anti-Sjögren's syndrome type B (anti-SSB) antibodies, on organ damage in patients with SLE.

\section{PATIENTS AND METHODS}

The study population consisted of 225 patients (37 males, 188 females; mean age $37.4 \pm 15$.9 years; range, 7 to 80 years) who were first diagnosed with SLE between August 2013 and November 2015 at Affiliated Hospital of Youjiang Medical University For Nationalities. All patients fulfilled the Systemic Lupus International Collaborating Clinics (SLICC) 2012 classification criteria for SLE. ${ }^{8}$ Patients suffering from rheumatoid arthritis, skin inflammation, systemic sclerosis, nodular polyarthritis, epilepsy, organic brain disease, psychosis, idiopathic thrombocytopenic purpura, primary glomerular disease and other diseases were excluded from the study. The study protocol was approved by the Affiliated Hospital of Youjiang Medical University For Nationalities Ethics Committee. A written informed consent was obtained from each patient. The study was conducted in accordance with the principles of the Declaration of Helsinki.

All patients were divided into either a positive or negative group according to the levels of serum antibodies. Correlations between the levels of serum antibody and renal damage, arthritis, photosensitivity, cheek erythema, neurologic disorders, hematological abnormalities and serosa inflammation were analyzed respectively.

The diagnostic indexes for renal disease were persistent proteinuria $>0.5 \mathrm{~g} /$ day or greater than $3+$ by dipstick and/or urine cellular casts including red blood cells, hemoglobin, granular, tubular or mixed. The diagnostic indexes for arthritis were non-erosive arthritis, involving two or more surrounding joints, characterized by swelling and pain in the joints. The diagnostic index for photosensitization was skin allergy caused by sunlight. The diagnostic index for malar rash was a flat or higher level of erythema in the buccal region than in the skin. The diagnostic indexes for neuropsychiatric disorders were convulsions (non-drug or metabolic disorders, such as uremia, ketoacidosis and electrolytes disorder) and mental symptoms (non-drug or metabolic disorder [as described above]). The diagnostic index for oral ulcers was a painless ulcer of the mouth or nasopharynx. The diagnostic indexes for hematology abnormality were hemolytic anemia with a granulophilocyte increase or when aleukocytosis decrease was detected to be lower than $4 \times 10^{9} / \mathrm{L}$ on two or more occasions. Lymphopenia was defined as $<1.5 \times 10^{9} / \mathrm{L}$ on two or more occasions and platelets count $<100 \times 10^{9} / \mathrm{L}$ without any other identifiable causes. The diagnostic indexes for serositis were pleuritis (chest pain, pleuralrale and pleural effusion) or pericarditis (abnormal electrocardiogram, pericardial rub or pericardial effusion).

Autoantibodies were analyzed by the Clinical Immunology Laboratory in the Affiliated Hospital of Youjiang Medical University For Nationalities. Peripheral blood samples were obtained from SLE. Fasting venous blood was obtained from all cases in the morning and serum was separated by centrifugation, and then stored in $-20^{\circ}$ to be measured. All the experimental procedures followed the manufacturer's recommended protocols, and the experimental reagents were purchased from EUROIMMUN Medical Laboratory Diagnostics Company, Lubeck, Germany. Immunoblotting was used for detection and the results were expressed as negative, suspicious positive, $1+, 2+$ and $3+$.

\section{Statistical analysis}

Statistical analysis of the data was performed using the IBM SPSS version 21.0 software (IBM Corp., Armonk, NY, USA). Chi-square test was used to compare the categorical data and t-test to compare the means between two groups. A t-test was applied when the test statistics followed a normal distribution. Data distribution was tested by the Shapiro-Wilk method. Two-sided $p<0.05$ was considered to be statistically significant.

\section{RESULTS}

The positive rates of seven types of autoantibodies were statistically significantly different in 225 specimens from all the SLE patients. The positive rate of anti-SSA antibodies 
Table 1. Comparison between anti-double stranded deoxyribonucleic acid antibody and organ damage

\begin{tabular}{|c|c|c|c|c|c|c|c|}
\hline Clinical manifestations & $+/-$ & Positive number & Positive rate & Negative number & Negative rate & $\chi^{2}$ value & $p$ \\
\hline \multirow{2}{*}{ Renal damage } & + & 90 & 40.00 & 76 & 33.78 & \multirow{2}{*}{9.803} & \multirow{2}{*}{0.002} \\
\hline & - & 18 & 80.00 & 41 & 18.22 & & \\
\hline \multirow{2}{*}{ Arthritis } & + & 60 & 26.67 & 70 & 31.11 & \multirow{2}{*}{0.420} & \multirow{2}{*}{0.517} \\
\hline & - & 48 & 21.33 & 47 & 20.89 & & \\
\hline \multirow{2}{*}{ Photosensitivity } & + & 10 & 4.44 & 24 & 10.67 & \multirow{2}{*}{5.544} & \multirow{2}{*}{0.019} \\
\hline & - & 98 & 43.56 & 93 & 41.33 & & \\
\hline \multirow{2}{*}{ Malar rash } & + & 30 & 13.33 & 43 & 19.11 & \multirow{2}{*}{2.064} & \multirow{2}{*}{0.151} \\
\hline & - & 78 & 34.67 & 74 & 32.89 & & \\
\hline \multirow{2}{*}{ Neuropsychiatric disorders } & + & 4 & 1.78 & 9 & 4.00 & \multirow{2}{*}{1.641} & \multirow{2}{*}{0.200} \\
\hline & - & 104 & 46.22 & 108 & 48.00 & & \\
\hline \multirow{2}{*}{ Oral ulcers } & + & 14 & 6.22 & 10 & 4.44 & \multirow{2}{*}{1.149} & \multirow{2}{*}{0.284} \\
\hline & - & 94 & 41.78 & 107 & 47.56 & & \\
\hline \multirow{2}{*}{ Hematological abnormality } & + & 91 & 40.44 & 81 & 36.00 & \multirow{2}{*}{7.044} & \multirow{2}{*}{0.008} \\
\hline & - & 17 & 7.56 & 36 & 16.00 & & \\
\hline \multirow{2}{*}{ Serositis } & + & 48 & 21.33 & 34 & 15.11 & \multirow{2}{*}{5.739} & \multirow{2}{*}{0.017} \\
\hline & - & 60 & 26.67 & 83 & 36.89 & & \\
\hline
\end{tabular}

was as high as 66.22\%, anti-dsDNA antibodies (48.0\%) was the second highest, followed by anti-Sm antibodies (41.78\%), anti-Rib antibodies (38.22\%), anti-U1RNP antibodies (36.0\%), AHAs (28.0\%), and anti-SSB antibodies (21.33\%).

Of these 225 patients, 166 patients had renal damage, 59 patients had no renal damage,
130 had arthritis, 95 had no arthritis, 34 were photosensitive, 191 were not photosensitive, 73 had malar rash, 152 had no malar rash, 13 had neuropsychiatric disorders, 212 had no neuropsychiatric lupus, 24 had oral ulcers, 201 had no oral ulcers, 149 had hematology abnormalities, 76 had no hematology abnormality, 82 had

Table 2. Comparison between anti-histone antibody and organ damage

\begin{tabular}{|c|c|c|c|c|c|c|c|}
\hline Clinical manifestations & $+/-$ & Positive number & Positive rate & Negative number & Negative rate & $\chi^{2}$ value & $p$ \\
\hline \multirow{2}{*}{ Renal damage } & + & 52 & 23.11 & 114 & 50.67 & \multirow{2}{*}{3.472} & \multirow{2}{*}{0.062} \\
\hline & - & 11 & 4.89 & 48 & 21.33 & & \\
\hline \multirow{2}{*}{ Arthritis } & + & 38 & 16.89 & 92 & 40.89 & \multirow{2}{*}{0.231} & \multirow{2}{*}{0.631} \\
\hline & - & 25 & 11.11 & 70 & 31.11 & & \\
\hline \multirow{2}{*}{ Photosensitivity } & + & 3 & 1.33 & 31 & 13.78 & \multirow{2}{*}{7.306} & \multirow{2}{*}{0.007} \\
\hline & - & 60 & 26.67 & 131 & 58.22 & & \\
\hline \multirow{2}{*}{ Malar rash } & + & 24 & 10.67 & 49 & 21.78 & \multirow{2}{*}{1.275} & \multirow{2}{*}{0.259} \\
\hline & - & 39 & 17.33 & 113 & 50.22 & & \\
\hline \multirow{2}{*}{ Neuropsychiatric disorders } & + & 1 & 0.44 & 12 & 5.33 & \multirow{2}{*}{1.855} & \multirow{2}{*}{0.173} \\
\hline & - & 62 & 27.56 & 150 & 66.67 & & \\
\hline \multirow{2}{*}{ Oral ulcers } & + & 8 & 3.56 & 16 & 7.11 & \multirow{2}{*}{0.379} & \multirow{2}{*}{0.538} \\
\hline & - & 55 & 24.44 & 146 & 64.89 & & \\
\hline \multirow{2}{*}{ Hematological abnormality } & + & 52 & 23.11 & 120 & 53.33 & \multirow{2}{*}{1.805} & \multirow{2}{*}{0.179} \\
\hline & - & 11 & 4.89 & 42 & 18.67 & & \\
\hline \multirow{2}{*}{ Serositis } & + & 20 & 8.89 & 62 & 27.56 & \multirow{2}{*}{0.834} & \multirow{2}{*}{0.361} \\
\hline & - & 43 & 19.11 & 100 & 44.44 & & \\
\hline
\end{tabular}


Table 3. Comparison between anti-U1 ribonucleoprotein antibody and organ damage

\begin{tabular}{|c|c|c|c|c|c|c|c|}
\hline Clinical manifestations & $+/-$ & Positive number & Positive rate & Negative number & Negative rate & $\chi^{2}$ value & $p$ \\
\hline \multirow{2}{*}{ Renal damage } & + & 66 & 29.33 & 100 & 44.44 & \multirow{2}{*}{3.882} & \multirow{2}{*}{0.049} \\
\hline & - & 15 & 6.67 & 44 & 19.56 & & \\
\hline \multirow{2}{*}{ Arthritis } & + & 50 & 26.67 & 80 & 31.11 & \multirow{2}{*}{0.810} & \multirow{2}{*}{0.368} \\
\hline & - & 31 & 13.78 & 64 & 28.44 & & \\
\hline \multirow{2}{*}{ Photosensitivity } & + & 17 & 7.56 & 17 & 7.56 & \multirow{2}{*}{3.407} & \multirow{2}{*}{0.065} \\
\hline & - & 64 & 28.44 & 127 & 56.44 & & \\
\hline \multirow{2}{*}{ Malar rash } & + & 27 & 12.00 & 46 & 20.44 & \multirow{2}{*}{0.046} & \multirow{2}{*}{0.831} \\
\hline & - & 54 & 24.00 & 98 & 43.56 & & \\
\hline \multirow{2}{*}{ Neuropsychiatric disorders } & + & 5 & 2.22 & 8 & 3.56 & \multirow{2}{*}{0.000} & \multirow{2}{*}{1.000} \\
\hline & - & 76 & 33.78 & 136 & 60.44 & & \\
\hline \multirow{2}{*}{ Oral ulcers } & + & 11 & 4.89 & 13 & 5.78 & \multirow{2}{*}{1.128} & \multirow{2}{*}{0.288} \\
\hline & - & 70 & 31.11 & 131 & 58.22 & & \\
\hline \multirow{2}{*}{ Hematological abnormality } & + & 69 & 30.67 & 103 & 45.78 & \multirow{2}{*}{1.110} & \multirow{2}{*}{0.292} \\
\hline & - & 12 & 5.33 & 41 & 18.22 & & \\
\hline \multirow{2}{*}{ Serositis } & + & 36 & 16.00 & 46 & 20.44 & \multirow{2}{*}{3.497} & \multirow{2}{*}{0.061} \\
\hline & - & 45 & 20.00 & 98 & 43.56 & & \\
\hline
\end{tabular}

serositis and 143 had no serositis. There were no statistically significant differences in the incidence of arthritis, malar rash, neuropsychiatric disorders and oral ulcers between anti-dsDNA antibody positive and negative groups. However, there were statistically significant differences among patients with renal damage, photosensitivity, hematology abnormalities and serositis $(\mathrm{p}<0.05)$ (Table 1$)$.
There were no statistically significant differences in the incidence of renal damage, arthritis, malar rash, neuropsychiatric disorders, oral ulcers, hematology abnormalities and serositis between AHA positive and negative groups ( $p>0.05)$; on the other hand, there were statistically significant differences among patients with photosensitivity $(\mathrm{p}<0.05)$ (Table 2).

Table 4. Comparison between anti-Smith antibody and organ damage

\begin{tabular}{|c|c|c|c|c|c|c|c|}
\hline Clinical manifestations & $+/-$ & Positive number & Positive rate & Negative number & Negative rate & $\chi^{2}$ value & $p$ \\
\hline \multirow{2}{*}{ Renal damage } & + & 86 & 38.22 & 80 & 35.56 & \multirow{2}{*}{26.179} & \multirow{2}{*}{$<0.001$} \\
\hline & - & 8 & 3.56 & 51 & 22.67 & & \\
\hline \multirow{2}{*}{ Arthritis } & + & 3 & 1.33 & 127 & 56.44 & \multirow{2}{*}{197.199} & \multirow{2}{*}{$<0.001$} \\
\hline & - & 91 & 40.44 & 4 & 1.78 & & \\
\hline \multirow{2}{*}{ Photosensitivity } & + & 20 & 8.89 & 14 & 6.22 & \multirow{2}{*}{4.784} & \multirow{2}{*}{0.029} \\
\hline & - & 74 & 32.89 & 117 & 52.00 & & \\
\hline \multirow{2}{*}{ Malar rash } & + & 29 & 12.89 & 44 & 19.56 & \multirow{2}{*}{0.187} & \multirow{2}{*}{0.665} \\
\hline & - & 65 & 28.89 & 87 & 38.67 & & \\
\hline \multirow{2}{*}{ Neuropsychiatric disorders } & + & 3 & 1.33 & 10 & 4.44 & \multirow{2}{*}{1.984} & \multirow{2}{*}{0.159} \\
\hline & - & 91 & 40.44 & 121 & 53.78 & & \\
\hline \multirow{2}{*}{ Oral ulcers } & + & 16 & 7.11 & 8 & 3.56 & \multirow{2}{*}{6.842} & \multirow{2}{*}{0.009} \\
\hline & - & 78 & 34.67 & 123 & 54.67 & & \\
\hline \multirow{2}{*}{ Hematological abnormality } & + & 80 & 35.56 & 92 & 40.89 & \multirow{2}{*}{6.727} & \multirow{2}{*}{0.009} \\
\hline & - & 14 & 6.22 & 39 & 17.33 & & \\
\hline \multirow{2}{*}{ Serositis } & + & 54 & 24.00 & 28 & 12.44 & \multirow{2}{*}{30.746} & \multirow{2}{*}{$<0.001$} \\
\hline & - & 40 & 17.78 & 103 & 45.78 & & \\
\hline
\end{tabular}


Table 5. Comparison between anti-ribosomal $\mathrm{P}$ protein antibody and organ damage

\begin{tabular}{lccccccc}
\hline Clinical manifestations & +- & Positive number & Positive rate & Negative number & Negative rate & $\chi^{2}$ value & $p$ \\
\hline \multirow{2}{*}{ Renal damage } & + & 75 & 33.33 & 91 & 40.44 & 16.663 & $<0.001$ \\
& - & 9 & 4.00 & 50 & 22.22 & & \\
Arthritis & + & 57 & 5.33 & 73 & 32.44 & 5.582 & 0.018 \\
& - & 27 & 12.00 & 68 & 30.22 & & \\
Photosensitivity & + & 18 & 8.00 & 16 & 7.11 & 4.170 & 0.041 \\
& - & 66 & 29.33 & 125 & 55.56 & & \\
Malar rash & + & 44 & 19.56 & 29 & 12.89 & 24.307 & $<0.001$ \\
& - & 40 & 17.78 & 112 & 49.78 & & \\
Neuropsychiatric disorders & + & 3 & 1.33 & 10 & 4.44 & 0.639 & 0.424 \\
& - & 81 & 36.00 & 131 & 58.22 & & \\
Oral ulcers & + & 12 & 5.33 & 12 & 5.33 & 1.842 & 0.175 \\
\multirow{4}{*}{ Hematological abnormality } & - & 72 & 32.00 & 129 & 57.33 & & \\
\multirow{2}{*}{ Serositis } & + & 79 & 35.11 & 93 & 41.33 & 23.067 & $<0.001$ \\
& - & 5 & 2.22 & 48 & 21.33 & & \\
& + & 42 & 18.67 & 40 & 17.78 & 10.634 & 0.001 \\
\hline
\end{tabular}

There were no statistically significant differences in the incidence of arthritis, photosensitivity, malar rash, neuropsychiatric disorders, oral ulcers, hematology abnormalities and serositis between anti-U1RNP antibody positive and negative groups ( $p>0.05)$; meanwhile, there were statistically significant differences among patients with renal damage $(\mathrm{p}<0.05)$ (Table 3).

There were no statistically significant differences in the incidence of malar rash and neuropsychiatric disorders between anti-Sm antibody positive and negative groups ( $p>0.05)$; however, there were statistically significant differences among patients with renal damage, arthritis, photosensitivity, oral ulcers, hematology abnormalities and serositis $(p<0.05)$ (Table 4).

There were no statistically significant differences in the incidence of neuropsychiatric disorders and oral ulcers between the anti-Rib antibody positive and negative groups ( $p>0.05$ ); on the other hand, there were statistically significant differences among patients with renal damage, arthritis, malar rash, photosensitivity, hematology abnormalities and serositis $(p<0.05)$ (Table 5).

Table 6. Comparison between antibodies, urine protein, and creatinine clearance rate

\begin{tabular}{|c|c|c|c|}
\hline \multirow[t]{2}{*}{ Antibody } & \multirow[t]{2}{*}{$+/-$} & 24-hour urinary protein (g) & \multirow{2}{*}{$\frac{\text { Creatinine clearance rate }(\mathrm{mL} / \mathrm{min})}{\text { Mean } \pm \mathrm{SD}}$} \\
\hline & & Mean \pm SD & \\
\hline \multirow{2}{*}{ Anti-dsDNA } & + & $2.2 \pm 1.6$ & $45.1 \pm 22.5^{*}$ \\
\hline & - & $2.7+2.3$ & $71.9 \pm 29.9$ \\
\hline \multirow{2}{*}{ Anti-U1RNP } & + & $2.3 \pm 1.6$ & $63.4+25.4$ \\
\hline & - & $2.5 \pm 2.2$ & $67.8 \pm 30.0$ \\
\hline \multirow{2}{*}{ Anti-Smith } & + & $2.4 \pm 1.6$ & $63.4+27.1$ \\
\hline & - & $2.4 \pm 2.5$ & $70.5 \pm 29.7$ \\
\hline \multirow{2}{*}{ Anti-ribosomal $\mathrm{P}$ protein } & + & $2.4 \pm 1.7$ & $63.4 \pm 27.1$ \\
\hline & - & $2.4 \pm 2.2$ & $69.1 \pm 30.5$ \\
\hline
\end{tabular}


Therewere no statistically significant differences in the incidence of renal damage, arthritis, malar rash, photosensitivity, neuropsychiatric disorders, oral ulcers, hematology abnormalities and serositis between the anti-SSA antibody positive and negative groups ( $p>0.05)$. And it was the same to anti-SSB antibody positive and negative groups ( $p>0.05)$.

Among the patients with renal damage, there was no statistically significant difference in the 24-hour urinary protein between the anti-dsDNA antibody positive and negative groups ( $p>0.05$ ). There was a statistically significant difference in the creatinine clearance rate between the anti-dsDNA antibody positive and negative groups $(p<0.05)$; however, there were no statistically significant differences in the 24-hour urinary protein excretion and creatinine clearance rate between the anti-U1RNP antibody, anti-Sm antibody and anti-Rib antibody positive and negative groups ( $p>0.05)$ (Table 6).

\section{DISCUSSION}

Systemic lupus erythematosus is an autoimmune disease characterized by multiple system and organ damage, and its clinical manifestations vary greatly from one individual to other. Because of the specific or relatively specific autoantibodies that may be found in the sera of patients with SLE, clinicians can diagnose the disease according to their autoantibodies. Anti-dsDNA antibody, AHA and extractable nuclear antigen (ENA) are the most common autoantibodies in SLE patients. ENA contains a variety of antibodies, such as anti-U1RNP, anti-Sm, anti-Rib, anti-SSA, anti-SSB, and anti-Scl-70 antibodies. This study aimed to determine the above autoantibodies and correlate them to SLE.

Anti-double stranded deoxyribonucleic acid antibody is only found in SLE patients and it is a high specific antibody of SLE. Several researchers have proven that anti-dsDNA antibodies correlate with the clinical manifestation, severity of illness, and disease activity and they may assist in assessing the condition and prognosis. ${ }^{9}$ It is generally accepted that renal damage usually occurs in active stage of disease, and anti-dsDNA antibodies were considered to play an important pathogenic role in that and to be a risk factor for lupus nephritis (LN). ${ }^{10}$ The results of this study showed that anti-dsDNA antibodies were associated with renal damage, photosensitization, hematological abnormality and serositis. Among the patients with renal damage, there was a significant decrease in creatinine clearance in patients with anti-dsDNA antibodies, suggesting that patients with anti-dsDNA antibodies are prone to renal damage and the extent of damage is more severe. In recent years, more and more studies have shown that anti-dsDNA antibodies had no correlation with renal damage and severity. ${ }^{11}$ Recent studies have found that $30 \%$ of LN patients have negative anti-dsDNA antibodies while $25 \%$ of SLE patients have positive antidsDNA antibodies but no LN occurrence. ${ }^{12}$ Therefore, more pathogenic antibodies and injury mechanisms of LN needed to be further studied. With more research in this field, it is becoming clear that nucleosomes and their antibody molecules appear to be more and more important in the pathogenesis of LN. $^{13}$ Napirei et al. ${ }^{14}$ found that the activity of anti-dsDNA antibodies disappeared after removal of nucleosomes and their antibody molecules. This suggests that nucleosomes and their antibody molecules play a bridging role in the process of the binding of antigens between the antibodies and the kidneys, and this may be the initiating antigen of LN pathological process. Therefore, the relationship between anti dsDNA antibody and renal damage needs to be further studied. Thus, we suggest that the severity of LN should be assessed based on the level of organ damage rather than the level of antibodies.

Histone is an alkaline protein, consisting of five subunits of $\mathrm{H}-1, \mathrm{H}-2 \mathrm{~A}, \mathrm{H}-2 \mathrm{~B}, \mathrm{H}-3$ and $\mathrm{H}-4$, and each subunit has its specific antibodies, collectively known as AHA. AHAs can appear in a variety of connective tissue diseases. In SLE, the positive rate is about $30 \% .{ }^{15}$ AHA can be detected in approximately $75 \%$ of drug-induced lupus patients as well as in idiopathic SLE patients. ${ }^{16}$ Regarding the specificity of this antibody to the diagnosis of SLE, different scholars have different views and even opposite conclusions. It is reported that AHAs are related to SLE renal damage and disease activity, and the specificity is better than the anti-dsDNA antibodies. ${ }^{17}$ 
Ribonucleoprotein antigens are sensitive to ribonucleotide enzyme and trypsin and are inactivated when heated. Therefore, only the U1 part can be precipitated, a type of ribonucleic acid proteins containing eight units, called antiU1RNP antibody. ${ }^{18}$ Anti-U1RNP antibody is an important serological basis for the diagnosis of mixed connective tissue disease (MCTD), and is the marker antibody for MCTD. ${ }^{19}$ In SLE, the positive rate is 30\% 40\%; however, the specificity is not high, and it may also be positive in the combination of other rheumatoid diseases. ${ }^{20}$ Studies have shown that the incidence of photosensitization, arthritis and renal damage in SLE patients with positive anti-U1RNP antibody is higher than that of negative patients. ${ }^{21}$ In this study, the positive rate was $36.0 \%$, and the incidence of renal damage was much higher in the anti-U1RNP antibody positive group, which was consistent with the relevant report. However, some scholars believe that patients with positive anti-U1RNP antibody often have negative antidsDNA antibody, and their kidneys are less affected..$^{22}$ Therefore, it is necessary to further study whether the anti-U1 RNP antibody is related to renal damage and its relevance.

Anti-Smith antibodies target at the level of small ribosomal proteins in the nucleus, and are almost always accompanied by RNP antibodies. The positive rate is 30\% 70\% in SLE patients. ${ }^{23}$ Although the detection rate is low (30\% 40\%), ${ }^{24}$ the specificity is as high as $92.2 \% \sim 99 \%$ and they are included in the diagnostic criteria of SLE. SmBB' and D are the substances that can react with anti-Sm antibody in the anti-Sm antigen. However, SmBB' and U1 RNP have the antigenic determinant, PPPGMRPP, which can cause crossreactions, and so $\mathrm{SmD}$ is considered to be the most specific Sm antigen in SLE. The most immunologically active protein in $\mathrm{SmD}$ antigens is SmD1, so anti-SmD1 antibody is considered to be a good marker antibody for SLE. ${ }^{25}$ As the patient's anti-Sm antibody continues to be positive, it is suitable for the diagnosis of early, atypical and anti-dsDNA antibody negative SLE cases, as well as a retrospective diagnostic index for SLE. ${ }^{26}$ Studies have shown that anti-Sm antibody is associated with arthritis, renal damage, oral ulcers and hematological abnormalities. In this study, the incidence of renal damage, arthritis and hematological abnormalities in SLE patients with positive anti-Sm antibodies was much higher, which was consistent with the reported results.

Anti-ribosomal $\mathrm{P}$ protein antibody (ARPA) is a type of antibody that mainly targets at the P0, P1 and P2 of the cytoplasm subunit phosphoric acid protein. The positive rate of patients with SLE was $14.0 \% \sim 22.0 \% .{ }^{27}$ Its specificity is high and the antibody positive patients often also present with nervous neurological damage. The anti-Rib antibody is often present during the duration of SLE activity, which is parallel to the abasement of dsDNA, and the difference is that the former will not disappear immediately with the remission of the disease, and will not be transferred for one year or two. It was found that the ARPA's positive rate and ARPA in cerebrospinal fluid were significantly higher than those in the control group. ${ }^{28}$ However, there are also reports that there is no association between ARPA and SLE neurological damage ${ }^{29}$ In this study, the incidence of renal damage, arthritis, photosensitivity, malar rash, hematological abnormalities and serositis in SLE patients with anti-Rib antibody positive group was much higher.

Researchers extracted and discovered SSA and SSB antigens in the blood of patients with Sjögren's syndrome (SS). There are proteins of molecular weights 52 and $60 \mathrm{KDa}$ in SSA antigen, so the anti-SSA antibody has the corresponding anti-52000 and anti-60000 anti-peptide antibodies, namely anti-Ro52 antibody and anti-Ro60 antibodies. ${ }^{30}$ It is reported that the positive rate of SSA/Ro antibodies in SLE patients is $30 \% \sim 50 \%, 95 \% \sim 100 \%$ in neonatal lupus and $60 \%$ in subacute cutaneous lupus erythematosus. ${ }^{31}$ The presence of only anti-Ro52 antibodies is very common in SS, while the presence of only anti-Ro60 antibodies is more common in SLE. The anti-SSB antibodies have the same significance as anti-SSA antibodies. But the anti-SSB antibodies positive rate is lower than anti-SSA antibodies, which are always accompanied by each other. Studies have shown that anti-SSA antibodies are associated with pulmonary hypertension, and anti-SSB antibodies are associated with hematological abnormalities. ${ }^{32}$ Anti-Scl-70 antibodies were mainly found in patients with systemic sclerosis. In this study, the positive detection rate was low (2.22\%); thus, no statistical analysis was performed. 
The main limitation of this study is the lack of any pathological diagnosis. Thus, further studies are needed based on renal biopsies to explore the predictive value of autoantibodies in the diagnosis and prognosis of SLE.

In conclusion, (i) anti-dsDNA, anti-U1RNP, anti-Sm and anti-Rib antibodies positive patients are more prone to renal damage; (ii) anti-Sm and anti-Rib antibodies positive patients are more vulnerable to arthritis; (iii) anti-dsDNA, antihistone, anti-Sm and anti-Rib antibodies positive patients are more prone to photosensitization; and (iv) anti-dsDNA, anti-Sm and anti-Rib antibodies positive patients are more prone to hematological abnormalities and serositis. Therefore, the detection of these autoantibodies can aid clinicians in assessing the organ damage in SLE patients and provide a powerful basis for early intervention and treatment while evaluating the severity and prognosis of the disease accordingly.

\section{Acknowledgments}

The authors appreciate the tutorials on statistical analysis from Professor Shusong Deng. The authors thank all participants involved in this study and they would also like to thank Dr. Dev Sooranna, Imperial College London, for editing the manuscript.

\section{Declaration of conflicting interests}

The authors declared no conflicts of interest with respect to the authorship and/or publication of this article.

\section{Funding}

This study was supported by grants from the National Natural Science Foundation of China, No. 81560271, Key Project of Scientific Research of the Guangxi Colleges and Universities, No. KY2015ZD092, and the Program of Natural Science Foundation of Guangxi, No. 2014GXNSFAA118253 and 2017GXNSFDA198005.

\section{REFERENCES}

1. Bakr A, Laimon W, El-Ziny MA, Hammad A, El-Hawary AK, Elsharkawy AA, et al. The emergence of systemic lupus erythematosus in hypothyroid patients: two case reports and mini review. Lupus 2014;23:825-8.

2. McCarty DJ, Manzi S, Medsger TA Jr, RamseyGoldman R, LaPorte RE, Kwoh CK. Incidence of systemic lupus erythematosus. Race and gender differences. Arthritis Rheum 1995;38:1260-70.
3. Alba P, Bento L, Cuadrado MJ, Karim Y, Tungekar MF, Abbs I, et al.Anti-dsDNA, anti-Sm antibodies, and the lupus anticoagulant: significant factors associated with lupus nephritis. Ann Rheum Dis 2003;62:556-60.

4. Isenberg DA, Garton M, Reichlin MW, Reichlin M. Long-term follow-up of autoantibody profiles in black female lupus patients and clinical comparison with Caucasian and Asian patients. Br J Rheumatol 1997;36:229-33.

5. Ravirajan CT, Rowse L, MacGowan JR, Isenberg DA. An analysis of clinical disease activity and nephritisassociated serum autoantibody profiles in patients with systemic lupus erythematosus: a cross-sectional study. Rheumatology (Oxford) 2001;40:1405-12.

6. Janwityanuchit $S$, Verasertniyom $\mathrm{O}$, Vanichapuntu M, Vatanasuk M.Anti-Sm: its predictive value in systemic lupus erythematosus. Clin Rheumatol 1993;12:350-3.

7. Winfield JB, Brunner CM, Koffler D. Serologic studies in patients with systemic lupus erythematosus and central nervous system dysfunction. Arthritis Rheum 1978;21:289-94.

8. Petri M, Orbai AM, Alarcón GS, Gordon C, Merrill JT, Fortin PR, et al. Derivation and validation of the Systemic Lupus International Collaborating Clinics classification criteria for systemic lupus erythematosus. Arthritis Rheum 2012;64:2677-86.

9. Zhao L, Jiang Z, Jiang Y, Ma N, Wang K, Zhang Y, et al. IL-22+CD4+ T-cells in patients with active systemic lupus erythematosus. Exp Biol Med (Maywood) 2013;238:193-9.

10. Im SR, Im SW, Chung HY, Pravinsagar P, Jang YJ. Cell- and nuclear-penetrating anti-dsDNA autoantibodies have multiple arginines in CDR3 of $\mathrm{VH}$ and increase cellular level of pERK and $\mathrm{Bcl}-2$ in mesangial cells. Mol Immunol 2015;67:377-87.

11. Ghirardello A, Doria A, Zampieri S, Tarricone E, Tozzoli R, Villalta D, et al. Antinucleosome antibodies in SLE: a two-year follow-up study of 101 patients. J Autoimmun 2004;22:235-40.

12. Jain D, Aggarwal HK, Kaverappa V, Dhayia S, Jain $P$, Yadav S. Anti-dsDNA negative and anti-Ro positive lupus nephritis: a report of a rare case. Reumatismo 2014;65:302-6.

13. Yang J, Xu Z, Sui M, Han J, Sun L, Jia X, et al. Co-Positivity for Anti-dsDNA, -Nucleosome and -Histone Antibodies in Lupus Nephritis Is Indicative of High Serum Levels and Severe Nephropathy. PLoS One 2015;10:0140441.

14. Napirei M, Karsunky H, Zevnik B, Stephan H, Mannherz HG, Möröy T. Features of systemic lupus erythematosus in Dnase1-deficient mice. Nat Genet 2000;25:177-81.

15. Sun XY, Shi J, Han L, Su Y, Li ZG. Anti-histones antibodies in systemic lupus erythematosus: prevalence and frequency in neuropsychiatric lupus. J Clin Lab Anal 2008;22:271-7. 
16. Katz U, Zandman-Goddard G.Drug-induced lupus: an update. Autoimmun Rev 2010;10:46-50.

17. van Bruggen $\mathrm{MC}$, Walgreen $\mathrm{B}$, Rijke TP, Tamboer W, Kramers K, Smeenk RJ, et al. Antigen specificity of anti-nuclear antibodies complexed to nucleosomes determines glomerular basement membrane binding in vivo. Eur J Immunol 1997;27:1564-9.

18. Migliorini $\mathrm{P}$, Baldini $\mathrm{C}$, Rocchi V, Bombardieri S. Anti-Sm and anti-RNP antibodies. Autoimmunity 2005;38:47-54.

19. Soltesz P, Bereczki D, Szodoray P, Magyar MT, Der H, Csipo I, et al. Endothelial cell markers reflecting endothelial cell dysfunction in patients with mixed connective tissue disease. Arthritis Res Ther 2010;12:78.

20. S L, Tony K, Raghupathy, V S, Malepati B. A rare case of mixed connective tissue disease (mctd) with intricate features of lupus, polymyositis and rheumatoid arthritis presenting with severe myositis. J Clin Diagn Res 2015;9:OD05-7.

21. Fredi M, Cavazzana I, Quinzanini M, Taraborelli M, Cartella S, Tincani A, et al. Rare autoantibodies to cellular antigens in systemic lupus erythematosus. Lupus 2014;23:672-7.

22. Miyamae T, Ito S, Machida H, Ozawa R, Higuchi R, Nakajima $\mathrm{S}$, et al. Clinical features and laboratory findings in children with both anti-dsDNA and antiU1-RNP antibody. Nihon Rinsho Meneki Gakkai Kaishi 2008;31:405-14.

23. Diallo MS, Mbengue $B$, Seck A, Ndao AC, Niang MS, Cissoko Y, et al. Evolution of autoantibodies profile in systemic lupus erythematosus according to age and clinical manifestations. Ann Biol Clin (Paris) 2014;72:351-8.

24. Chan EY, Mok TM, Lawton JW, Ko OK, Ho L, Lau CS. Comparison of counter immunoelectrophoresis with immunoblotting for detection of anti-extractable nuclear antigen antibodies in systemic lupus erythematosus.
Asian Pac J Allergy Immunol 1999;17:275-9.

25. Mahler M, Fritzler MJ, Blüthner M. Identification of a SmD3 epitope with a single symmetrical dimethylation of an arginine residue as a specific target of a subpopulation of anti-Sm antibodies. Arthritis Res Ther 2005;7:19-29.

26. Flechsig A, Rose T, Barkhudarova F, Strauss R, Klotsche J, Dähnrich $\mathrm{C}$, et al. What is the clinical significance of anti-Sm antibodies in systemic lupus erythematosus? A comparison with antidsDNA antibodies and C3. Clin Exp Rheumatol 2017;35:598-606.

27. Pradhan V, Patwardhan M, Rajadhyaksha A, Dhawale N, Ghosh K. Neuropsychiatric manifestations and associated autoantibodies in systemic lupus erythematosus patients from Western India. Rheumatol Int 2015;35:541-5.

28. Hirohata S, Arinuma Y, Takayama M, Yoshio T. Association of cerebrospinal fluid anti-ribosomal $\mathrm{p}$ protein antibodies with diffuse psychiatric/ neuropsychological syndromes in systemic lupus erythematosus. Arthritis Res Ther 2007;9:44.

29. Pasoto SG, Viana VS, Bonfa E. The clinical utility of anti-ribosomal $\mathrm{P}$ autoantibodies in systemic lupus erythematosus. Expert Rev Clin Immunol 2014;10:1493-503.

30. Peene I, Meheus L, De Keyser S, Humbel R, Veys EM, De Keyser F. Anti-Ro52 reactivity is an independent and additional serum marker in connective tissue disease. Ann Rheum Dis 2002;61:929-33.

31. Boh EE. Neonatal lupus erythematosus. Clin Dermatol 2004;22:125-8.

32. Li J, Leng X, Li Z, Ye Z, Li C, Li X, et al. Chinese SLE treatment and research group registry: III. association of autoantibodies with clinical manifestations in Chinese patients with systemic lupus erythematosus. J Immunol Res 2014;2014:809389. 